\title{
The effect of female arrivals on mate monopolization in the yellow dung fly
}

Received: 4 October 2002 / Revised: 11 February 2003 / Accepted: 14 February 2003 / Published online: 25 March 2003 (C) Springer-Verlag 2003

\begin{abstract}
The degree of resource monopolization relates to the distribution of resources in space and time. In general, monopolization is predicted to be high when resources (food or mates) are clumped in space, dispersed in time, and predictable in space or time. Using the yellow dung fly, Scathophaga stercoraria (Diptera: Scathophagidae), we qualitatively tested a general model that predicts the distribution of mating success among competing males based on the temporal pattern of female arrivals relative to mating time and a ranking of males in priority of access to the resource (here by body size). In a laboratory experiment approximating the natural mating situation, a constant number of males of various sizes were allowed to compete for females. As predicted, mate monopolization decreased as the temporal clumping of female arrivals increased, mediated by either a decrease in the mean or an increase in the variance of female interarrival times, which were manipulated independently. Males appeared to adjust their behavior to variation in female arrivals in a manner consistent with the marginal value theory of Parker and associates: forcible take-overs of females were rarer, and copula durations shorter, when females arrived regularly at short intervals. Therefore, a complex interaction of variation in intrinsic characteristics affecting male resource holding potential, mating time and stochastic, extrinsic variables increasing temporal clumping of mates generally reduces the variance in mating success among competing males and thus ultimately the opportunity and intensity of sexual selection on traits influencing male success. This theory extends operational sex ratio theory at the mechanistic, behavioral level.
\end{abstract}

Communicated by L. Simmons

W. U. Blanckenhorn (৫) · J. Frei · M. Birrer

Zoologisches Museum,

Universität Zürich,

Winterthurerstrasse 190, 8057 Zürich, Switzerland

e-mail: wolfman@zoolmus.unizh.ch

Tel.: +41-1-6354972

Fax: +41-1-6356818
Keywords Mating system - Operational sex ratio . Resource monopolization - Scathophaga stercoraria . Sexual selection

\section{Introduction}

Resource monopolization is one of the prime mechanisms increasing the variance in fitness among individuals. The degree of resource monopolization has been related to the distribution of resources in space and time (Emlen and Oring 1977). For example, when the environmental potential for the monopolization of mates is high, competition for mates will be strong, leading to extreme variation in mating success and intense sexual selection (Emlen and Oring 1977; Thornhill and Alcock 1983; Arnold and Duvall 1994). In general, resource defense and monopolization are predicted to be high when resources are clumped in space, dispersed in time, and predictable in space or time. This applies to competition for mates and food (Brown 1964; Emlen and Oring 1977; Warner 1980; Blanckenhorn 1991; Grant et al. 1995, 2000; Blanckenhorn et al. 1998). An individual's fitness therefore generally depends on the interaction of intrinsic factors that determine its quality or resource holding potential (e.g. body size) and extrinsic factors affecting resource availability.

In resource defense or lek mating systems, multiple males wait and compete for incoming females. Ever since Emlen and Oring's (1977) seminal paper, the operational sex ratio (OSR: number of females/number of males competing at the mating site) has become a key parameter in mating system studies because it strongly affects the strength of sexual selection (Arnold and Wade 1984; Andersson 1994, p. 149ff; Arnold and Duvall 1994). However, while the OSR is regularly estimated in phenomenological field studies of sexual selection (e.g. Andersson 1994, p. 149; Jann et al. 2000), experimental manipulations to test specific predictions of OSR theory at the mechanistic, behavioral level are comparatively scarce (e.g. Alonso-Pimentel and Papaj 1996; Verell and 
Krenz 1998; Sirot and Brockmann 2001). This is particularly true for the effects of temporal environmental variability on the OSR and its generalization, the competitor-to-resource ratio (Blanckenhorn 1991; Grant and Kramer 1992; Bryant and Grant 1995; Grant et al. 1995, 2000; Blanckenhorn et al. 1998). Moreover, the OSR is an incomplete and thus inexact measure in at least two ways. First, because in mating aggregations mates typically arrive and leave continuously over time, the OSR is merely (the average of) an instantaneous estimate of a dynamic parameter that can fluctuate considerably. Second, at any point in time a considerable proportion of individuals present at the mating site may not be actually competing for mates. This is because mating can include courtship, copulation and mate guarding, and thus last a considerable time. By analogy to the physiological or life history "time-out" required to produce a new batch of gametes (cf. Clutton-Brock and Parker 1992), males currently engaged in mating cannot compete for a newly arriving female, thus effectively increasing the OSR.

In a competitive mating situation, the best male (by whatever criteria) is expected to obtain most mates. As just outlined, a male's ability to monopolize females, and hence the variance in mating success among competing males, will crucially depend on the interaction between the temporal pattern of female arrival rates and the time it takes to mate (Grant et al. 1995; Blanckenhorn et al. 1998). That is, temporal clumping, and thus ultimately the OSR, has to be defined relative to mating or, in general, handling time. This was formalized in an analytical queuing model that generates predictions about the distribution of resources among competitors based on the temporal patterns of resource arrival, the handling time of the resource, and a ranking of competitors in priority of access to the resource (Blanckenhorn 1991; Blanckenhorn and Caraco 1992). In a mating context, the model qualitatively predicts a decrease in the variance in mating success among competitors if: (1) the average inter-arrival time of mates decreases (i.e. mate availability increases); (2) the average mating time increases; and (3) the variance in mate arrivals or mating time increases, provided that the average mating time exceeds the average inter-arrival time (otherwise the converse prediction results; Blanckenhorn and Caraco 1992). Under some simplifying assumptions, quantitative predictions are possible (Blanckenhorn et al. 1998). In general, it is easier to manipulate resource (mate) arrivals, as the handling (mating) time is primarily determined by the animals. Nevertheless, mating time, i.e. copula duration, itself often depends on the physiological state and environmental circumstances, most notably the probability of obtaining another mate (Parker 1970c; Parker and Simmons 1994; summarized in Simmons 2001, p. 198ff). The same holds true for the handling time of a food item (Stephens and Krebs 1986; e.g. Blanckenhorn and Viele 1999).

In this study, we provide the second test of Blanckenhorn and Caraco's (1992) model in a mating context, using the yellow dung fly Scathophaga stercoraria (sometimes Scatophaga: Diptera: Scathophagidae). The yellow dung fly is a classic model system for studies of sexual selection and conflict (Parker 1979). It is common on cow pastures in north-temperate Europe. Multiple males soon arrive at freshly deposited dung pats to wait for females ready to lay their eggs into the dung. Females usually arrive singly in a temporally dispersed manner (Parker 1970a; Reuter et al. 1998). As males are larger, they can seize females without courtship and force copulation, and females have few behavioral means to choose or reject males. Pre-copulatory sexual selection is therefore primarily mediated by male-male competition (Parker 1979), and the OSR is typically highly male biased (Jann et al. 2000). As in many species (Andersson 1994), large males enjoy a mating advantage (Borgia 1981; Jann et al. 2000). Copulation usually takes place on the dung or in the surrounding grass and takes about 35 min (Parker 1970b, 1970c). During the ensuing oviposition, which lasts 10-20 min, the male guards the female against other competitors (Parker 1970b). In our laboratory experiments, we simulated this situation in a semi-natural setting. A constant number of males of various sizes were allowed to compete for females. We independently manipulated both the mean and variance in arrival times of females, observing the mating behavior and success of males in relation to their body size rank. We predicted that the degree of mate monopolization by large males, i.e. the variance in mating success among all males, would decrease when female arrivals were more temporally clumped (Blanckenhorn and Caraco 1992; Blanckenhorn et al. 1998).

\section{Methods}

We used either laboratory-reared or field-collected S. stercoraria of various ages and sizes from our study population in Fehraltorf near Zurich. Before and after use in this experiment, individuals were held singly, at $20^{\circ} \mathrm{C}$, in $100 \mathrm{ml}$ bottles containing water, sugar (for energy) and ad libitum Drosophila melanogaster as prey (for the production of eggs and sperm: Foster 1967).

For the experiment, we assembled groups of eight males into well-aerated transparent plastic boxes approximately $15 \times 15 \times 25 \mathrm{~cm}$ in size. The boxes had a screen door on one side and contained the above nutrients (however during the experimental trials no Drosophila). For each experimental trial, we introduced a small dung pat, approximately $12 \mathrm{~cm}$ in diameter, on a large piece of filter paper. (Fresh dung was collected in Fehraltorf and subsequently stored frozen for several weeks before it was defrosted and used in this experiment.) In nature, dung pats tend to be larger, although small pats are also common, e.g. when cows walk while defecating. Eight males corresponds roughly to the average number of males found on pats of similar size at Fehraltorf, where yellow dung fly males have been found to be ideal-free distributed with regard to females but small pats tend to be overused by both sexes (see Fig. 3 in Blanckenhorn et al. 2000). The males were chosen randomly, except that we assured (by eye) a wide range of body sizes. Their exact body size (rank) was not known until it was estimated using hind tibia length after the experiment. For individual identification, all males were marked with dots of enamel paint on their thorax. Seventeen groups were tested over a period of several weeks. Typically, groups stayed together for up to 5 days, with each group undergoing three treatments (explained below), in randomized order, on one of these days. At the end of the 
Mean \& Variance

$\infty 0000$

○ 0

\section{0}

○ (3) High \& High

- (2) High \& Low

(1) Low \& Low

\section{$\begin{array}{llllllll}0 & 25 & 50 & 75 & 100 & 125 & 150 & 175\end{array}$ \\ Inter-arrival time (min)}

Fig. 1 Schedule of female inter-arrival times for the three treatments

experiment, the groups were disbanded and individuals later measured. Each male was used only once.

We always added eight females ready to lay eggs per experimental trial. We independently manipulated the mean (low $=5 \mathrm{~min}$ or $\mathrm{high}=25 \mathrm{~min}$ ) and the variance (low=0 or high $=1,000)$ in inter-arrival times. Hence, each group of males experienced three treatments: (1) low mean/low variance, (2) high mean/low variance and (3) high mean/high variance (Fig. 1). These numbers were somewhat arbitrary and perhaps extreme, albeit certainly within the range observed in nature. Parker (1970a; see also Fig. 1 in Reuter et al. 1998) reported that females arrive at average intervals of 4-5 min soon after dung deposition, with female arrivals becoming increasingly rarer as the dung pat ages. In the field, the OSR ranges from 0.05-0.6 (Parker 1970d; Jann et al. 2000; Kraushaar and Blanckenhorn 2002). Depending on the time interval at which it was estimated, the OSR of our treatments ranged from $1 / 8=0.125$ (instantaneous) to $8 / 8=1$ (over the whole trial). Intuitively, treatment 1 corresponds to the highest and treatment 2 to the lowest OSR $(1>3>2)$. Both inter-arrival means used in our experiment were less than the average copulation duration of yellow dung flies of about 35 min (Parker 1970b, 1970c; Parker and Simmons 1994). We thus predicted the degree of mate monopolization by large males (i.e. the variance in male mating success within groups) to be greatest in treatment 2 and smallest in treatment 1 ( $2>3>1$; cf. Introduction).

When a female entered the box, we observed which male mated with her and, if possible, measured the duration of the ensuing copulation to the nearest minute. When post-copulatory guarding occurred, this was noted but its duration not measured. We also noted if and when a male took over a female from another before copulation was completed. The probability of such a take-over ranges between 6\% and 25\% in nature (Parker 1970d; Borgia 1980; Sigurjónsdóttir and Parker 1981). If in our experiment a take-over occurred during copulation (but not thereafter), the female was scored as having been captured by the second male, as in this species the second male to mate fathers the majority of the offspring (Parker 1970c; Simmons 2001). As the probability of a take-over depends on opportunity, we predicted it to follow the same ranking of treatments as above $(2>3>1)$. After a female finished oviposition and was released by the male, or (more frequently) just before the end of female oviposition, the female or the guarding pair were carefully removed from the box (and the male immediately returned) to prevent a second mating with that female. In general, this was possible without much disturbance of the mating behavior.

\section{Results}

We analyzed the total number of matings each male obtained per trial (maximum possible range: $0-8$; actual

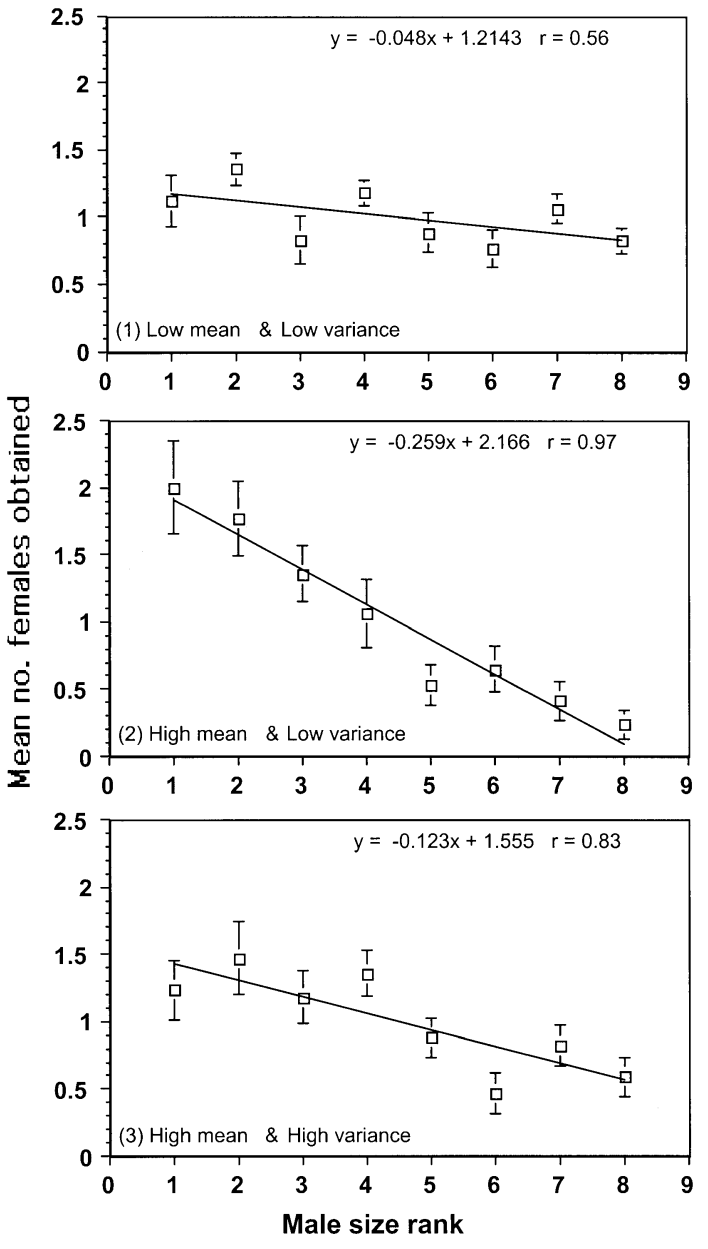

Fig. 2 Mean \pm SE number of females obtained as a function of male size rank for three treatments varying in female inter-arrival time mean and variance ( $n=17$ groups)

range $0-4)$ as a function of treatment and male size rank (1-8). We used doubly repeated-measures (treatment and male size rank) ANOVA, because the groups were our experimental units, each group received each treatment, and the eight males within each group were not statistically independent. Overall, the number of females obtained decreased, as expected, with male size rank $\left(F_{7,112}=5.30, \quad P<0.001 ;\right.$ Fig. 2$)$. More crucially, the treatment by male size rank interaction was also significant $\left(F_{14,224}=2.74, P=0.001\right)$, showing that the large male mating advantage varied among the three treatments as predicted: largest in treatment 2 and smallest in treatment 1 (Fig. 2). This interaction was also significant in our a priori planned comparisons: when testing for the effect of an increase in mean inter-arrival time from 5-25 min (with variance $=0$ held constant; treatment 1 vs 2 : $F_{7,112}=3.96, P=0.001$ ), and when testing for the effect of an increase in inter-arrival time variance (with mean $=25$ min held constant; treatment 2 vs 3 : $\left.F_{7,112}=2.46, P=0.022\right)$. There was no treatment main effect because the total number of females delivered in each treatment was constant. 


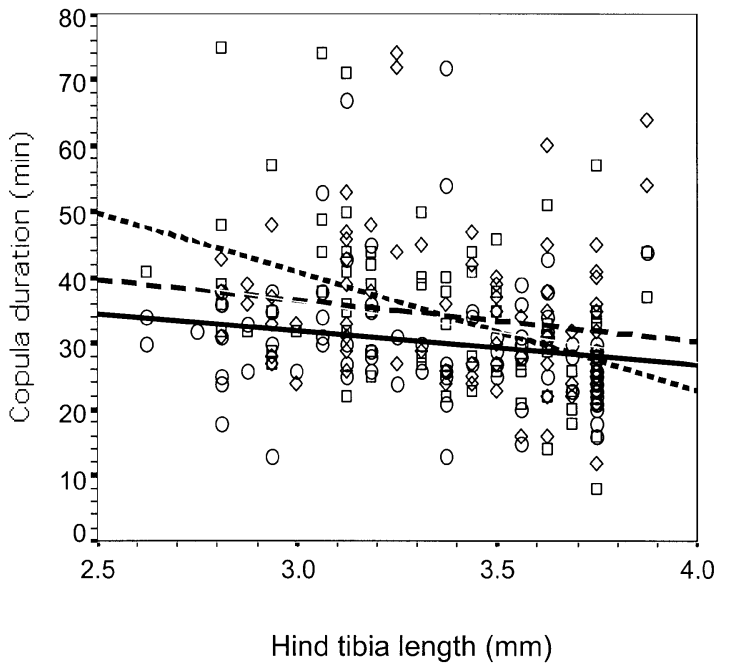

Fig. 3 Copula duration as a function of male size for three treatments varying in female arrival rate mean and variance: treatment 1 (frequent and regular; circles and solid line), treatment 2 (infrequent and regular; diamonds and broken line) and treatment 3 (infrequent and irregular; squares and dotted line; $n=271$ undisturbed copulations)

Of a total of 408 possible copulations to be observed in all groups and treatments, we obtained data on 335 copulations (the others were not observed completely). The proportion of take-overs varied with treatment $\left(\chi_{1}^{2}=5.86, \quad P=0.016\right)$ and among groups $\left(\chi^{2}{ }_{1}=6.27\right.$, $P=0.002$; interaction $P>0.2$; binary logistic regression): the fewest take-overs occurred in treatment 1 (12 of 99 copulations: $12.1 \%$ ), an intermediate number in treatment 2 (22 of 114:19.3\%) and most in treatment 3 (30 of 122: $24.6 \%$ ). The same analysis additionally revealed that the probability of suffering a take-over was greater for smaller males (negative effect of tibia length: $\chi^{2}{ }_{1}=18.87, P<0.001$; all interactions $P>0.2$ ): in 50 of 64 cases of a male take-over the female was larger than the defender.

As usual in this species (e.g. Parker and Simmons 1994), copula duration decreased with male size (overall $r=-0.28, \quad F_{1,265}=23.21, P<0.001$; ANCOVA on $n=271$ undisturbed copulations only; Fig. 3). Additionally, this relationship (treatment by male size interaction: $F_{2,265}=$ 4.26, $P=0.015)$ and copula duration itself $\left(F_{2,265}=4.78\right.$, $P=0.009)$ changed with treatment: $30.05 \pm 1.04 \mathrm{~min}$ $($ mean \pm SE) and partial $r=-0.17$ for treatment 1 , $33.84 \pm 1.16 \mathrm{~min}$ and partial $r=-0.17$ for treatment 2 , and $33.97 \pm 1.27 \mathrm{~min}$ and partial $r=-0.47$ for treatment 3 (Fig. 3). An analogous analysis showed that copula duration also decreased with the number of previous copulations (actual range: $0-3$; overall $r=-0.15, F_{1,265}=$ 5.90, $P=0.016$ ), independent of treatment (treatment effect and interaction both $P>0.2$ ). Post-copulatory guarding occurred after $65 \%$ of all observed copulations, equally for all treatments (treatment, group and body size effects $P>0.2$; binary logistic regression), but its duration was not measured.

\section{Discussion}

As predicted by a general theoretical model (Blanckenhorn and Caraco 1992), mate monopolization in the yellow dung fly decreased as the temporal clumping of female arrivals increased. This confirms earlier work with a few species (water striders, zebra fish and Japanese medaka) on the effects of temporal clumping on the monopolization of food (Blanckenhorn 1991; Grant and Kramer 1992; Bryant and Grant 1995) and mates (Grant et al. 1995; Blanckenhorn et al. 1998), thus highlighting the generality and fundamental nature of the underlying processes. Temporal clumping results both from faster arrivals of more females over a given period of time (a decrease in mean inter-arrival times; treatment 1 relative to treatment 2) and greater variance in arrivals given the same mean (treatment 3 relative to treatment 2). Whether resource arrivals are temporally clumped depends crucially on the time it takes to process the resource, i.e. handling time (here mating time; Blanckenhorn 1991; Blanckenhorn and Caraco 1992). Thus the scaling of resource arrivals and handling time, which are stochastic in nature, is crucial for predicting the degree of monopolization within groups. In general, the reduction in mate monopolization by synchronous mate arrivals will have stronger effects in species with longer mating times (i.e. courtship plus copulation plus guarding).

The consequence of this interaction of mate arrivals and mating time is always the same: when females arrive more synchronously, males with greater resource holding potential (here larger males) obtain fewer mates (and smaller males obtain more mates) than expected if their intrinsic characteristics alone affected mating success. This reduces variance in mating success among competitors (cf. Bateman 1948; Sutherland 1985) and hence ultimately the intensity of sexual selection on male traits (Arnold and Wade 1984; Arnold and Duvall 1994). In nature, numerous yellow dung fly males arrive early and synchronously at the mating site, a freshly deposited cow dung pat, whereas fewer females arrive later and more temporally dispersed (Parker 1970a, 1970b). Consequently, the OSR at the mating site (Emlen and Oring 1977) is highly male biased (Blanckenhorn et al. 2000; Jann et al. 2000). This dispersed female arrival pattern has been interpreted as an indirect mechanism of female choice increasing the likelihood of mating with a superior, larger male (Reuter et al. 1998). Variation in female arrivals, though typically not directly measured, thus inevitably contributes to the (stochastic) variation in large male mating advantage and the intensity of sexual selection on male body size observed for $S$. stercoraria in the field (Jann et al. 2000; Kraushaar and Blanckenhorn 2002). This means that merely assessing the OSR is insufficient, or at least incomplete.

Despite a wealth of experimental studies on yellow dung fly mating, this is the first study allowing groups of males to compete for females in a semi-natural setting. Males in our experiment appeared to adjust their behavior to variation in female arrivals. First, the mean copula 
duration observed here, ranging from 30-34 min, was very close to that observed by Parker and associates for single matings in the laboratory (range 30-39 min; Parker 1970c; Ward and Simmons 1991; Parker et al. 1993; Parker and Simmons 1994, 2000). Nevertheless, in our experiment copula duration was shortest in treatment 1 , when females arrived at short intervals. This is consistent with the marginal value theory of Parker and associates, which predicts that males should shorten copulations when the probability of obtaining another mate increases (summarized in Simmons 2001, p. 198ff). Our finding that smaller males copulate for longer, a common result in yellow dung fly studies, is also consistent with this theory, as larger males in general have a greater probability of obtaining more mates (Parker and Simmons 1994; Simmons 2001). Mate guarding prolongs the overall mating time and thus effectively increases temporal clumping. It is thus conceivable that males, in addition to copula duration, also curtailed guarding duration when females arrived in a temporally clumped manner. However, we did not measure guarding duration in this study, but only whether guarding occurred or not. This was the case in about $65 \%$ of all cases, irrespective of treatment. Second, the take-over rates were lowest in treatment 1 , when females arrived regularly at short intervals. As this was the treatment resulting in the lowest variance in mating success among competitors, this can be easily explained by lack of opportunity, as there were fewer males not involved in mating at any given time. However, we can offer no explanation for the result that take-overs were most frequent in treatment 3, rather than in treatment 2 in which there was most opportunity, except perhaps that the degree of female clumping was not so different in these two treatments (Fig. 1). The high overall take-over rate of about $19 \%$ found here can at least partly be explained by the confined setting, in which flies could not easily escape usurpation (cf. Parker 1970d; Borgia 1980; Sigurjónsdóttir and Parker 1981). As expected, due to their greater resource holding potential (Sigurjónsdóttir and Parker 1981; Parker and Simmons 1994), males taking over a female were larger than the defender in a great majority of cases. Moreover, Parker and Simmons (1994) show theoretically that a change in the take-over rate should in turn influence the optimal copula duration: if take-overs become more important for mate acquisition, large males will disproportionately gain time to acquire new mates, and the relationship between male size and (optimal) copula duration should become steeper, exactly as we found for treatment 3 (Fig. 3). Therefore, both our copula duration and our take-over results are entirely consistent with the marginal value theory of Parker and associates.

In summary, our experimental manipulation qualitatively confirmed a theoretical model predicting decreased mate monopolization with increasing temporal clumping of mate arrivals (Blanckenhorn 1991; Blanckenhorn and Caraco 1992). This results from a complex interaction of variation in intrinsic characteristics affecting male quality or resource holding potential (e.g. body size) and often stochastic, extrinsic (i.e. environmental) variables affecting mate arrivals and handling. This theory extends OSR theory at the mechanistic, behavioral level (cf. Grant et al's. 2000 concept of the competitor-to-resource ratio). Its generality has now been experimentally demonstrated in three different species in a foraging (Blanckenhorn 1991; Grant and Kramer 1992; Bryant and Grant 1995; Blanckenhorn et al. 1998; Grant et al. 2000) as well as a mating context (Grant et al. 1995; Blanckenhorn et al. 1998; this study). In general, temporal clumping of mates reduces variance in mating success among competing males (cf. Bateman 1948; Sutherland 1985) and thus ultimately the opportunity and intensity of sexual selection on traits influencing male success (Arnold and Wade 1984; Arnold and Duvall 1994). Particularly in male dominated mating systems, male control of mating opportunities may thus be reduced by female behavior directly or indirectly affecting the synchrony of mate availability, in turn ultimately (re)structuring the mating system in their favor (cf. Reuter et al. 1998; Nunn 1999).

Acknowledgements We thank the Swiss National Fund for financial support. This experiment was performed by students of the behavioral biology course 2001 at the University of Zürich.

\section{References}

Alonso-Pimentel H, Papaj DR (1996) Operational sex ratio versus gender density as determinants of copulation duration in the walnut fly, Rhagoletis juglandis. Behav Ecol Sociobiol 39:171180

Andersson M (1994) Sexual selection. Princeton University Press, Princeton

Arnold SJ, Duvall D (1994) Animal mating systems: a synthesis based on selection theory. Am Nat 143:317-348

Arnold SJ, Wade MJ (1984) On the measurement of natural and sexual selection: theory and applications. Evolution 38:709734

Bateman AJ (1948) Intra-sexual selection in Drosophila. Heredity $2: 349-368$

Blanckenhorn WU (1991) Foraging in groups of water striders: effects of variability in handling time and prey arrivals. Behav Ecol Sociobiol 28:221-226

Blanckenhorn WU, Caraco T (1992) Social subordinance and a resource queue. Am Nat 139:442-449

Blanckenhorn WU, Viele STN (1999) Foraging in yellow dung flies: testing for a small male time budget advantage. Ecol Entomol 24:1-6

Blanckenhorn WU, Grant JWA, Fairbairn DJ (1998) Monopolization in a resource queue: water striders competing for food and mates. Behav Ecol Sociobiol 42:63-70

Blanckenhorn WU, Morf C, Reuter M (2000) Are dung flies idealfree distributed at their mating and oviposition sites? Behaviour 137:233-245

Borgia G (1980) Sexual competition in Scatophaga stercoraria: size- and density related changes in male ability to capture females. Behaviour 75:185-206

Borgia G (1981) Mate selection in the fly Scatophaga stercoraria: female choice in a male-controlled system. Anim Behav 29:7180

Brown JL (1964) The evolution of diversity in avian territorial systems. Wilson Bull 76:160-169

Bryant MJ, Grant JWA (1995) Resource defence, monopolization and variation of fitness in groups of female Japanese medaka 
depend on the synchrony of food arrival. Anim Behav 49:14691479

Clutton-Brock TH, Parker GA (1992) Potential reproductive rates and the operation of sexual selection. Q Rev Biol 67:437-455

Emlen ST, Oring LW (1977) Ecology, sexual selection, and the evolution of mating systems. Science 197:215-223

Foster W (1967) Hormone-mediated nutritional control of sexual behavior in male dung flies. Science 158:1596-1597

Grant JWA, Kramer DL (1992) Temporal clumping of food arrival reduces its monopolization and defence by zebra fish, Brachydanio rerio. Anim Behav 44:101-110

Grant JWA, Bryant MJ, Soos CE (1995) Operational sex ratio, mediated by synchrony of female arrival, alters the variance of male mating success in Japanese medaka. Anim Behav 49:367375

Grant JWA, Gaboury C, Levitt HL (2000) Competitor-to-resource ratio, a general formulation of operational sex ratio, as a predictor of competitive aggression in Japanese medaka. Behav Ecol 11:670-675

Jann P, Blanckenhorn WU, Ward PI (2000) Temporal and microspatial variation in the intensities of natural and sexual selection in the yellow dung fly Scathophaga stercoraria. J Evol Biol 13:927-938

Kraushaar U, Blanckenhorn WU (2002) Population variation in sexual selection and its effect on body size allometry in two species of flies with contrasting sexual size dimorphism. Evolution 56:307-321

Nunn CL (1999) The number of males in primate social groups: a comparative test of the socioecological model. Behav Ecol Sociobiol 46:1-13

Parker GA (1970a) The reproductive behaviour and the nature of sexual selection in Scatophaga stercoraria (Diptera: Scatophagidae). V. The female's behaviour at the oviposition site. Behaviour 37:140-168

Parker GA (1970b) The reproductive behaviour and the nature of sexual selection in Scatophaga stercoraria (Diptera: Scatophagidae). II. The fertilisation rate and the spatial and temporal relationships of each sex around the site of mating and oviposition. J Anim Ecol 39:205-228

Parker GA (1970c) Sperm competition and its evolutionary effect on copula duration in the fly Scatophaga stercoraria (Diptera: Scatophagidae). J Insect Physiol 16:1301-1328

Parker GA (1970d) The reproductive behaviour and the nature of sexual selection in Scatophaga stercoraria L. (Diptera:
Scathophagidae). IV. Epigamic recognition and competition between males for the possession of females. Behaviour 37:113-139.

Parker GA (1979) Sexual selection and sexual conflict. In: Blum NS, Blum NA (eds) Sexual selection and reproductive competition in insects. Academic Press, New York, pp 123-166

Parker GA, Simmons LW (1994) Evolution of phenotypic optima and copula duration in dungflies. Nature 370:53-56

Parker GA, Simmons LW (2000) Optimal copula duration in yellow dung flies: ejaculatory duct dimensions and sizedependent sperm displacement. Evolution 54:924-935

Parker GA, Simmons LW, Ward PI (1993) Optimal copula duration in dungflies: effects of frequency dependence and female mating status. Behav Ecol Sociobiol 32:157-166

Reuter M, Ward PI, Blanckenhorn WU (1998) An ESS treatment of the pattern of female arrival at the mating site in the yellow dung fly Scathophaga stercoraria. J Theor Biol 195:363-370

Sigurjónsdóttir H, Parker GA (1981) Dung fly struggles: evidence for assessment strategy. Behav Ecol Sociobiol 8:219-230

Simmons LW (2001) Sperm competition and its evolutionary consequences in the insects. Princeton University Press, Princeton

Sirot LK, Brockmann HJ (2001) Costs of sexual interactions to females in Rambur's forktail damselfly, Ischnura ramburi. Anim Behav 61:415-424

Stephens DW, Krebs JR (1986) Foraging theory. Princeton University Press, Princeton

Sutherland WJ (1985) Chance can produce a sex difference in variance in mating success and explain Bateman's data. Anim Behav 33:1349-1352

Thornhill R, Alcock J (1983) The Evolution of insect mating systems. Harvard University Press, Cambridge

Verell PA, Krenz JD (1998) Competition for mates in the mole salamander, Ambystoma talpoideum: tactics that may maximize male mating success. Behaviour 135:121-138

Ward PI, Simmons LW (1991) Copula duration and testes size in the yellow dung fly, Scathophaga stercoraria (L.): the effects of diet, body size, and mating history. Behav Ecol Sociobiol 29:77-85

Warner RR (1980) The coevolution of behavioral and life-history characteristics. In: Barlow GW, Silverberg J (eds) Sociobiology: beyond nature/nurture? Westview, Boulder, Colo., pp $151-188$ 